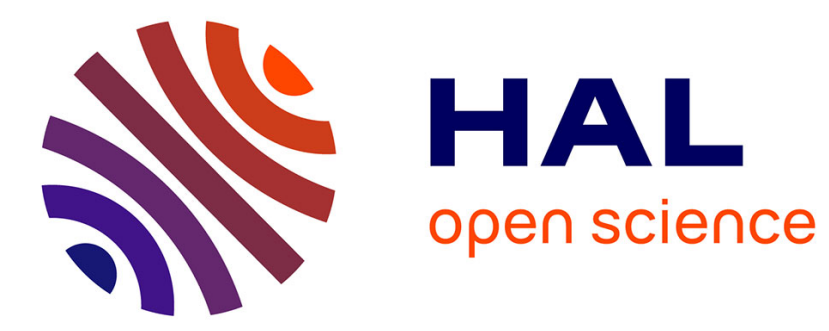

\title{
Resonant Acoustic Propagation and Negative Density in Liquid Foams
}

\author{
Juliette Pierre, Benjamin Dollet, Valentin Leroy
}

\section{To cite this version:}

Juliette Pierre, Benjamin Dollet, Valentin Leroy. Resonant Acoustic Propagation and Negative Density in Liquid Foams. Physical Review Letters, 2014, 112 (14), pp.148307. 10.1103/PhysRevLett.112.148307 . hal-01127796v3

\section{HAL Id: hal-01127796 \\ https://hal.science/hal-01127796v3}

Submitted on 9 Mar 2015

HAL is a multi-disciplinary open access archive for the deposit and dissemination of scientific research documents, whether they are published or not. The documents may come from teaching and research institutions in France or abroad, or from public or private research centers.
L'archive ouverte pluridisciplinaire HAL, est destinée au dépôt et à la diffusion de documents scientifiques de niveau recherche, publiés ou non, émanant des établissements d'enseignement et de recherche français ou étrangers, des laboratoires publics ou privés. 


\title{
Resonant Acoustic Propagation and Negative Density in Liquid Foams
}

\author{
Juliette Pierre, ${ }^{1}$ Benjamin Dollet, ${ }^{2}$ and Valentin Leroy ${ }^{1}$ \\ ${ }^{1}$ Laboratoire Matière et Systèmes Complexes, Université Paris-Diderot, CNRS (UMR 7057), Paris, France \\ ${ }^{2}$ Institut de Physique de Rennes, Université Rennes 1, CNRS (UMR 6251), Rennes, France
}

(Dated: February 24, 2014)

\begin{abstract}
We measured the dispersion relation for acoustic longitudinal waves in liquid foams, over a broad frequency range $(60-600 \mathrm{kHz})$. Strong dispersion was found, with two non-dispersive behaviors, separated by a negative density regime. A new model, based on the coupled displacements of films, liquid channels and gas in the foam, rationalizes all the experimental findings.
\end{abstract}

Liquid foams, dispersions of gas bubbles in a liquid matrix stabilized by surfactants [1], are present in a wide range of industrial applications, from food and personal care to ore flotation and enhanced oil recovery [2]. They are opaque materials, hence difficult to characterize in depth, and new ways of probing them are highly desirable. Acoustic probes are good candidates, since they are unexpensive and nonintrusive, and widely used as such in nondestructive testing [3]. However, surprisingly little is known about liquid foam acoustics, contrary to other multiphasic media such as porous media [4], colloidal suspensions, or emulsions $[5,6]$. Most of the few existing experimental studies reported speeds of sound of order $50 \mathrm{~m} / \mathrm{s}$ [7-9], close to the so-called Wood model [10]. The latter treats foams as an effective medium, which density and compressibility are given by the mixture law, i.e. averages of those of the gas and liquid phases, weighted by their respective volume fractions. However, much higher speeds of sound, of order $200 \mathrm{~m} / \mathrm{s}$, were also measured $[11,12]$, and some studies reported a resonant behavior $[13,14]$, reminiscent of the low frequency resonance of a single bubble in an unbounded liquid, the so-called Minnaert resonance [15], which is the key ingredient of acoustic propagation through dilute bubbly liquids $[16,17]$. In this Letter, we use a novel setup [18] to measure speed of sound and attenuation through liquid foams over a large range of frequencies $f(60-600 \mathrm{kHz})$ and bubble radii $(15-50 \mu \mathrm{m})$. Our results are explained by a new model, which fully reconciles all aforementioned different viewpoints on liquid foam acoustics. In particular, we show that liquid foams are natural acoustic metamaterials, exhibiting a negative effective density over a large range of frequencies and bubble sizes.

The general principle of our technique is to measure the complex transmission of short ultrasonic pulses through a foam sample of known thickness. From this complex transmission, we can determine the effective complex wavevector as a function of frequency: $k=\omega / v+\mathrm{i} \alpha$, with $\omega=2 \pi f$, and $v$ and $\alpha$ the phase velocity and attenuation of sound. In an ageing foam, the bubble median radius $R$ increases over time by gas diffusion between bubbles [1]. Hence, making measurements at different times, we obtain $k(\omega, R)$, the complex wavevector as a function of frequency and median radius. Some aspects of the ultrasonic technique and the foam production and characterization are briefly described below; more details can be found in [18] for the former, and [19] for the latter.

Transmission measurements were performed with two broadband air transducers. The thickness of the foam samples was set to $0.5 \mathrm{~mm}$ by sandwiching them between two plastic films, which were as thin as possible to allow a good transmission of ultrasound. An inversion procedure was used to deduce the effective wavevector $k$ of sound in the foam from the complex transmission measured through the three-layer system \{film-foamfilm $\}$. This procedure relied on the assumption that either the effective density $\rho_{\text {eff }}$ or the effective compressibility $\chi_{\text {eff }}$ were known. Guided by the model presented below, we assumed that the effective compressibility of the liquid foam was given by the usual mixture law: $\chi_{\text {eff }}=\Phi \chi_{\ell}+(1-\Phi) \chi_{g}$, where $\Phi$ is the liquid volume fraction and $\chi_{\ell, g}$ the compressibility of the liquid and gaseous phase.

Liquid foams with liquid volume fractions ranging from $3 \%$ to $22 \%$ were obtained by the two-syringe method [19]. As in [19], the foaming liquid was an aqueous solution containing $10 \mathrm{~g} / \mathrm{L}$ of sodium dodecyl sulfate to ensure good foamability, and $0.5 \mathrm{~g} / \mathrm{L}$ of xanthane to reduce drainage. Given the small thickness of the samples $(0.5 \mathrm{~mm})$, the typical radius of the bubbles (less than $50 \mu \mathrm{m})$ and the presence of xanthane, we were able to neglect the gradient of liquid fraction due to gravity [20]. The gaseous phase was air saturated with vapor of $\mathrm{C}_{6} \mathrm{~F}_{14}$, an insoluble gas that slows down ageing. Thus, the typical time over which the foam was changing ( $~ 10$ minutes) was long enough not only to consider the acoustic measurements as instantaneous, but also to perform bubble size measurements [19]. To do that, we took a small sub-sample of the foam from the syringe, and pour it on a liquid bath to obtain a bubble raft, a two-dimensional structure easy to image. Assuming that the left-over in the syringe was ageing the same way as the foam between the films, we were able to follow the time evolution of the bubble size distribution. We found lognormal distributions $\exp \left\{-[\ln (r / R)]^{2} / 2 \epsilon^{2}\right\} /(r \epsilon \sqrt{2 \pi})$ with a median radius $R$ going from 15 to $50 \mu \mathrm{m}$ in 90 minutes, and a polydispersity of $\epsilon \simeq 0.4$ without significant time evolution (Fig. 1c). 


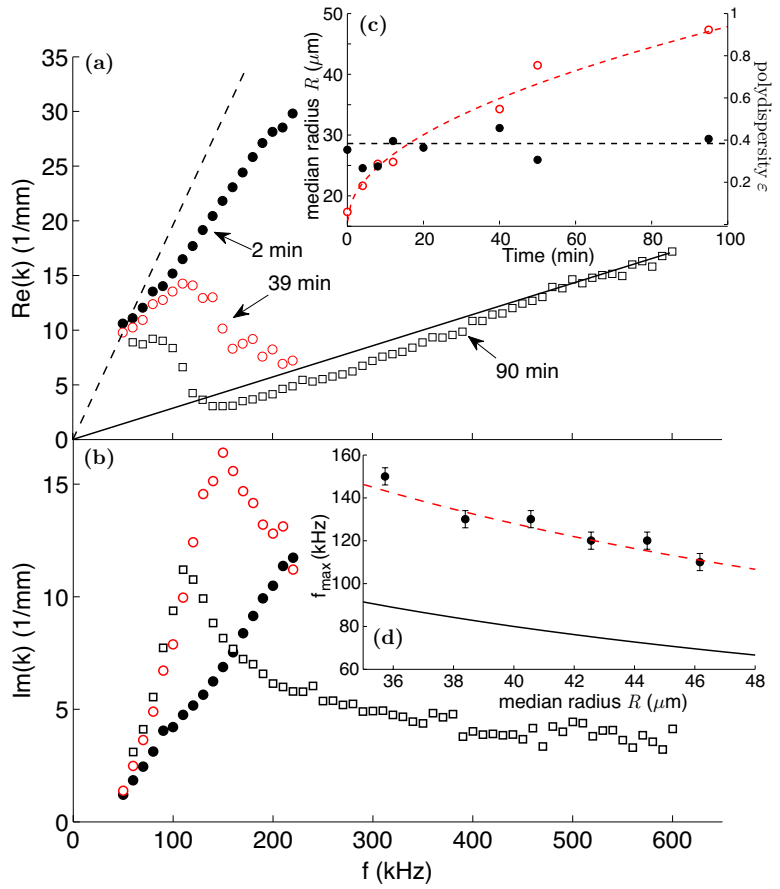

FIG. 1: (a) Real part of the wavenumber as a function of the frequency, measured at three different ageing times in a liquid foam with $\Phi=11 \%$. Lines show dispersion curves for constant velocities of $32 \mathrm{~m} / \mathrm{s}$ (dash) and $220 \mathrm{~m} / \mathrm{s}$ (solid). (b) Imaginary part of the wavenumber. (c) Median radius of the bubbles (o) and polydispersity $(\bullet)$ as functions of the ageing time. (d) Frequency of the maximum of attenuation as a function of the median radius; solid line is the Minnaert frequency $f_{M}$, dashed line is $1.6 f_{M}$.

Measurements shown in Fig. 1a clearly indicate that the propagation of acoustic waves in a liquid foam is dispersive. The phase velocity at low frequencies is close to the value predicted by Wood law: $v=\left[\left(\Phi \rho_{\ell}+(1-\right.\right.$ $\left.\left.\Phi) \rho_{g}\right)\left(\Phi \chi_{\ell}+(1-\Phi) \chi_{g}\right)\right]^{-1 / 2}=32 \mathrm{~m} / \mathrm{s}$ for $\Phi=11 \%$. But it significantly deviates from this value as the frequency increases and the foam ages. At $t=90 \mathrm{~min}$ the phase velocity is of the order of $220 \mathrm{~m} / \mathrm{s}$.

The attenuation (Fig. 1b) is also frequency-dependent and evolves as the foam ages. For the longest times (i.e. largest bubbles), a peak is clearly visible. One can report the frequency of the maximum of attenuation $f_{\max }$ as a function of $R$ (Fig. 1d), and compare it with the Minnaert frequency $f_{M}=\sqrt{3 \gamma P_{0} / \rho} /(2 \pi R)$ [15], where $P_{0}$ is the pressure of the gas in the bubbles, and $\gamma$ the ratio of the specific heat capacities. Taking $f_{\max }=1.6 f_{M}$ gives a reasonable law (dashed line in Fig. 1d), suggesting an effective Minnaert resonance, as proposed in previous experiments $[13,14]$. However, the Minnaert frequency is calculated for a single bubble in an infinite volume of liquid of density $\rho$, and for highly concentrated media such as foams, it seems unrealistic. Replacing $\rho$ by the actual density of the surrounding medium $\Phi \rho$ would lead to $3 f_{M}$ for $\Phi=11 \%$, larger than the 1.6 factor we measured. Moreover, it predicts a resonance frequency that decreases with $\Phi$, contrary to our experiments (Fig. 2). Finally, the range of radii over which we measured a maximum of attenuation (Fig. 1d) is too small to validate a scaling law in $f_{\max } \sim 1 / R$. Instead of limiting our analysis to the peak of attenuation, we can take advantage of all the radii by collapsing the data on a master curve: we plot $k R^{p}$ as a function of $f R^{p}$, with an exponent $p$. As shown in Fig 3, a good collapse is obtained for $p=1.5$, as suggested by the model we present below. This indi-

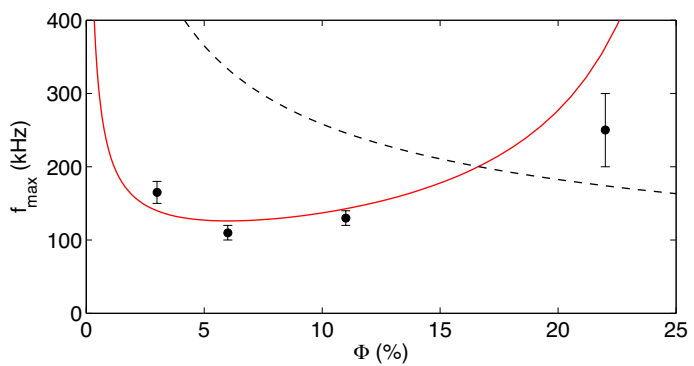

FIG. 2: Frequency of the maximum of attenuation as a function of the liquid fraction, for median radii of $40 \mu \mathrm{m}$. Dashed line is the Minnaert resonance frequency with an effective density: $f_{M} / \sqrt{\Phi}$. Solid line is the prediction of our model.

cates that $R$ is the key parameter that governs the frequency dependence of the acoustic behavior of the foam (the polydispersity being almost constant with time).

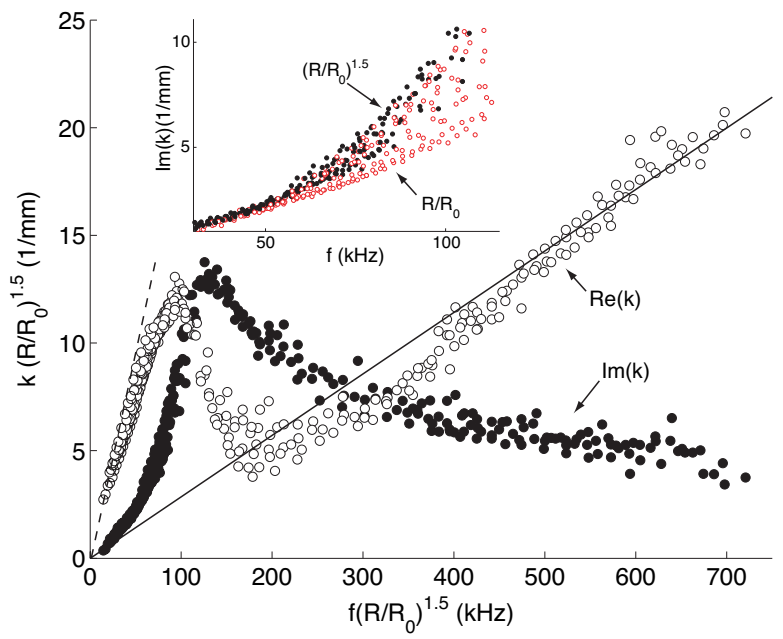

FIG. 3: Rescaled dispersion relation for a reference median radius of $R_{0}=40 \mu \mathrm{m}$. Lines are for constant velocities of 32 (dash) and $220 \mathrm{~m} / \mathrm{s}$ (solid). Inset: the $R^{-1.5}$ scaling (solid symbols) is better than the $R^{-1}$ one (open symbols).

Contrary to usual bubbly media, bubbles in a foam are in contact through thin films, separated by liquid channels (also called Plateau borders) and vertices [1]. Usually films contain a very small amount of water, 
hence films and liquid channels have very different inertia, whose influence we shall now model. In first approximation, we neglect polydispersity, and we idealize the foam as a one-dimensional array of spatial periodicity $d$ of the following unit cell (Fig. 4a): a flexible, circular membrane of thickness $e$ and radius $a$ (representing the soap film), attached to a rigid ring of external radius $b$ and mass $m_{c}$ (representing the liquid channel), and surrounded by air of density $\rho_{a}$. Experiments show that the wavelength $\lambda=2 \pi / \operatorname{Re}(k)$ remains larger than $7 R$, even at the highest frequency investigated (Fig. 3). Hence, the model is based on the hypothesis $\lambda \gg d$.

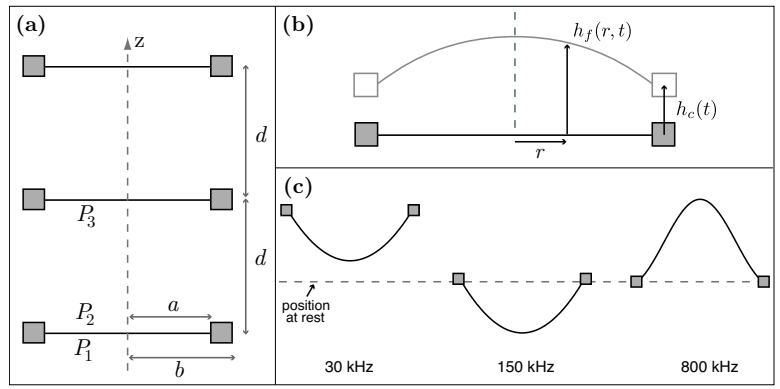

FIG. 4: (a) Sketch of the idealized foam structure: a periodic array of flexible films and rigid rings (representing the liquid channels), separated by a distance $d$. (b) Zoom on one unit cell: the flexible film is attached to a rigid ring sketched as a square. (c) Profiles of a $20 \mu \mathrm{m}$ radius film at three different frequencies, as predicted by the model for a $\Delta P=1 \mathrm{~Pa}$ excitation. Note that the deformations are not as high as suggested by the picture: the maximum of displacement of the film at $800 \mathrm{kHz}$ for instance is about $1.5 \mathrm{~nm}$, i.e. more than four orders of magnitude smaller than the diameter of the film.

To predict the acoustic wavevector, we need to model the behavior of the unit cell under compression (effective compressibility $\chi_{\text {eff }}$ ), as well as its motion driven by the average pressure gradient $\left(P_{3}-P_{1}\right) / d$ (effective density $\left.\rho_{\text {eff }}\right): k^{2}=\omega^{2} \rho_{\text {eff }} \chi_{\text {eff }}$. Our model geometry resembles that of Lee et al. [21], who studied sound propagation through an array of elastic membranes. In particular, in the limit $\lambda \gg d$, the effective compressibility is the same: an almost uniform pressure change is applied to the unit cell, hence its total volume changes as the sum of the liquid volume change and the gas volume change, which yields the usual mixture law for the effective compressibility.

The effective density, on the other hand, is more complicated because the flexible film and the rigid ring do not react the same way when submitted to a given pressure gradient. A portion of the film between $r$ and $r+\mathrm{d} r$ is driven by the pressure force $2 \pi r \mathrm{~d} r\left(P_{1}-P_{2}\right)$ (see Fig. 4a) and by the vertical projection of the tension force $4 \pi \sigma\left[\left(r \partial h_{f} / \partial r\right)_{r+\mathrm{dr}}-\left(r \partial h_{f} / \partial r\right)_{r}\right]$. In harmonic regime, $h_{f}(r, t)=\operatorname{Re}\left[z_{f}(r) \mathrm{e}^{-\mathrm{i} \omega t}\right]$, the profile of the film is thus given by $z_{f}(r)=A J_{0}(q r)+\Delta P /\left(\rho e \omega^{2}\right)$, where $A$ is its amplitude of vibration, $\Delta P=P_{2}-P_{1}$ the driving pressure, $q=\omega \sqrt{\rho e / 2 \sigma}$ the wavenumber of capillary waves on the film [? ], and $J_{0}$ the Bessel function of order 0 of the first kind [22]. The unknown constant $A$ is determined by matching the motion of the film edge with that of the ring: $h_{f}(r=a, t)=h_{c}(t)$. It is interesting to note that even though the pressure field is uniform in the lateral direction, it produces oscillations of the film. This is due to the film being attached to the ring, which has a different inertia. The ring is driven by the pressure difference $\Delta P$ and by the traction of the film $-4 \pi \sigma a(1-\mathrm{i} \omega \tau)\left(\partial z_{f} / \partial r\right)_{r=a}$, in which we include a phenomenological damping time $\tau$ to account for a friction force (see SM1 for a full discussion on dissipation). We finally get the full profile

$$
z_{f}(r)=\frac{\Delta P}{\rho e \omega^{2}}\left[1-\frac{m_{c}-m_{f}\left(b^{2}-a^{2}\right) / a^{2}}{m_{c}+m_{f}(1-\mathrm{i} \omega \tau) \mathcal{H}(q a)} \frac{J_{0}(q r)}{J_{0}(q a)}\right]
$$

where $m_{f}=\pi a^{2} e \rho$ is the mass of the film and $\mathcal{H}(q a)=$ $2 J_{1}(q a) /\left[q a J_{0}(q a)\right]$.

We now consider the motion of air between two consecutive films, which is driven by the pressure difference $P_{2}-P_{3}$. As $\lambda \gg d$, we may consider that the whole air of the unit cell moves as one block with displacement $z_{a}$ given by $-m_{a} \omega^{2} z_{a}=\left(P_{2}-P_{3}\right) \pi b^{2}$, where $m_{a}$ is the mass of the air in the cell. Moreover, the air in contact with the film and ring is also entrained by their motion. By continuity of displacement, we simply assume that $z_{a}$ equals the average displacement of the film and ring: $z_{a}=(1-x) z_{c}+\frac{2 x}{a^{2}} \int_{0}^{a} r z_{f}(r) \mathrm{d} r$, where $x=a^{2} / b^{2}$ is the fraction of the surface covered by the film. We can then calculate the average displacement $z_{a}$ of the unit cell, and relate it to the effective density $\rho_{\text {eff }}$ by $\omega^{2} \rho_{\mathrm{eff}} z_{a}=\left(P_{3}-P_{1}\right) / d$. Finally, we obtain an effective density of the foam $\rho_{\text {eff }}=(1-\Phi) \rho_{a}+\Phi^{\prime} \rho$, where the frequency-dependent effective liquid volume fraction $\Phi^{\prime}$ is given by

$$
\Phi^{\prime}=\frac{\Phi_{c}+\Phi_{f}(1-\mathrm{i} \omega \tau) \mathcal{H}(q a)}{1+\left(x^{2} \frac{\Phi_{f}+\Phi_{c}}{\Phi_{f}}-2 x\right)[1-\mathcal{H}(q a)]-\mathrm{i} \omega \tau x \mathcal{H}(q a)}
$$

with $\Phi_{c}$ and $\Phi_{f}$ the volume fraction of liquid contained in the channels and in the films, respectively: $\Phi_{c}+\Phi_{f}=\Phi$. Eq. (2) predicts two asymptotic non-dispersive behaviors. At low frequencies, $\mathcal{H}(q a) \simeq 1$, hence $\operatorname{Re} \Phi^{\prime}=\Phi$ : the mixture law for $\rho_{\text {eff }}$ is recovered. At high frequencies, $\mathcal{H}(q a)$ tends towards 0 , leading to $\operatorname{Re}\left(1 / \Phi^{\prime}\right)=$ $(1-x)^{2} / \Phi_{c}+x^{2} / \Phi_{f}$. If $\Phi_{f} \ll \Phi_{c}[x /(1-x)]^{2}$ (i.e. films contain a negligible part of water, but with a nonnegligible surface) this last formula reduces to $x^{2} / \Phi_{f}$ : the effective liquid density is governed by the films, as in Kann's model [12].

The transition between the two asymptotic regimes can be inspected by noting that $1-\mathcal{H}(q a) \sim_{0}-(q a)^{2} / 8$. Thus, within the approximation $\Phi_{c} \gg \Phi_{f}|1-2 / x|$, 
Eq. (2) becomes $\Phi^{\prime} \simeq \Phi_{c} /\left[1-\left(\omega / \omega_{0}\right)^{2}-\mathrm{i} x \omega \tau\right]$, similar to the response function of an harmonic oscillator with a resonance frequency $\omega_{0}^{2}=16 \sigma \Phi_{f} /\left(\rho a^{2} e x^{2} \Phi\right)$ and a damping factor $x \omega \tau$. Interestingly, this resonance frequency depends neither on $a$ nor on $e$ since $\Phi_{f}=n_{f} \pi a^{2} e, n_{f}$ being the number per unit volume of films perpendicular to the direction of propagation. If we consider that there are $N$ such films per bubble, we obtain $n_{f}=3 N(1-\Phi) /\left(4 \pi R^{3}\right)$ and the resonance frequency becomes

$$
\omega_{0}^{2}=\frac{12 N \sigma(1-\Phi)}{x^{2} \rho \Phi R^{3}}
$$

which predicts the experimentally observed $R^{-1.5}$ scaling law. The mechanism of this resonance is different from Minnaert's one: inertia also comes from water, but here the restoring force is due to the tension of the film, not the compressibility of air.

To go beyond and get a quantitative prediction over the full range of frequencies and sizes, we refine the model and include polydispersity, as explained in SM1. Briefly, this amounts to changing $\mathcal{H}(q a)$ by $\mathcal{I}=$ $\int \mathcal{H}(q a) a^{2} n(a) \mathrm{d} a / \int a^{2} n(a) \mathrm{d} a$ in the expression (2) of the effective liquid fraction, with $n(a)$ the distribution of film radii. If we assume that this distribution is lognormal with a median radius $a_{0}$, a polydispersity $\epsilon_{f}$ and a total number of films per unit volume $n_{f}$, the model counts a total of nine parameters. Three are known: $\sigma=35 \mathrm{mN} / \mathrm{m}, \Phi_{c}=11 \%, \epsilon=0.4$. Four can be estimated: $x, n_{f}, a_{0}$ and $\epsilon_{f}$. The surface fraction covered by films in a liquid foam was studied by Princen [24]. He found the following empirical dependence on $\Phi$ : $x=1-3.20[7.70+(1-\Phi) / \Phi]^{-1 / 2}$, which gives $x=0.2$ in our case. Then one can estimate that $n_{f}=1620$ films per $\mathrm{mm}^{3}$ (assuming $N=1$ ), $a_{0}=15 \mu \mathrm{m}$ (taking an average of 6 films per bubble imposes $\left.x=1.5\left(a_{0} / R\right)^{2}\right)$ and $\epsilon_{f}=0.4$ (same polydispersity as for bubble radii). The two remaining parameters were fitted to the experimental data: $\tau$ governs the width of the resonance, $e$ the high-frequency effective density. With $\tau=10 \mu \mathrm{s}$ and $e=70 \mathrm{~nm}$, the model agrees well with the experimental data (Fig. 5). This value of $e$ is compatible with the usual measurements of film thickness, of a few tens of nanometers [1]. It also justifies the Taylor expansion leading to Eq. (3), since it leads to $(q a)^{2} \simeq 0.15$ at the resonance frequency $(130 \mathrm{kHz})$. It is less easy to compare $\tau$ to any standard measurement, and the local study of the microscopic dissipation mechanisms at high frequency is left as a perspective of our work.

The model also captures well the dependence on $\Phi$ of the frequency of the maximum of attenuation, as shown in Fig. 2. The polydispersity has to be included to obtain a quantitative comparison (see SM1), but Eq. (3) is sufficient to understand the non-monotonic behavior: for an increasing liquid fraction, the increase of density $(1 / \sqrt{\Phi}$ term, as in the modified-Minnaert relation) is dominated by the shrinkage of the films $(1 / x$ term).
A salient feature of our study is the existence of a negative real part of the density over an extended range of frequencies (100 to $300 \mathrm{kHz}$ ). The mechanism of this negative effective density is well illustrated by looking at the displacement of a film and a liquid channel predicted by (1) (see Fig. 4c). At low frequencies, they move in phase with the incoming pressure. At high frequencies, only the film moves. At intermediate frequencies, while the liquid channel has a small in-phase displacement, the film moves out of phase with an amplitude large enough to compensate for its small inertia. The net average movement of the system is then out of phase, which leads to a negative effective density [?].

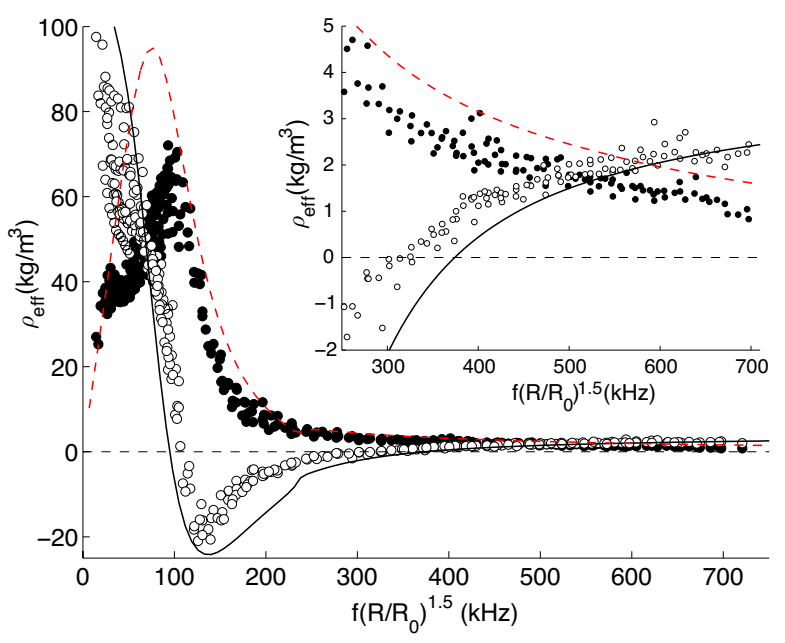

FIG. 5: Comparison between the measured effective complex density ( $\circ$ real part, $\bullet$ imaginary part) and prediction of the equivalent of Eq. (2) in the polydisperse model (see SM1).

As a conclusion, we have evidenced two regimes for the sound propagation in a liquid foam, separated by a resonance. Our study reconciles the seemingly contradictory results previously reported: a low speed of sound, compatible with Wood's model, at low frequency and small bubble size; a much higher one, slightly lower than that in air, at high frequency and large bubble size; and a resonance in between, with a maximum of attenuation. This rich variety of behaviors is fully captured by a model coupling the motion of air, films and liquid channels.

Finally, we have shown for the first time that liquid foams are naturally acoustic single-negative metamaterials, with an effective negative density over an extended range of frequencies and bubble sizes. Moreover, in contrast with most existing metamaterials $[25,26]$, which are synthetic, highly ordered materials, this property exists even though the foam is isotropic and polydisperse. Our findings could thus bring fresh insight to design metamaterials for applications such as acoustic insulation.

We acknowledge funding support from the Agence Nationale de la Recherche (ANR-11-BS09-001). We thank 
J.-C. Bacri, C. Gay, F. Graner and A. Saint-Jalmes for fruitful discussions.

[1] I. Cantat, S. Cohen-Addad, F. Elias, F. Graner, R. Höhler, O. Pitois, F. Rouyer, A. Saint-Jalmes, Foams, edited by S. J. Cox, Oxford University Press (2013).

[2] Foam Engineering: Fundamentals and Applications, edited by P. Stevenson, Wiley (2012).

[3] J. Blitz, G. Simpson, Ultrasonic Methods of Nondestructive Testing, Chapman \& Hall (1996).

[4] K. Attenborough, Physics Reports 82, 179 (1982).

[5] A. S. Dukhin, P. J. Goetz, Adv. Colloid Interface Sci. 92, $72(2001)$

[6] R. E. Challis, M. J. W. Powey, M. L. Mather, A. K. Holmes, Rep. Prog. Phys. 68, 1541 (2005).

[7] I. I. Goldfarb, I. R. Shreiber, F. I. Vafina, J. Acoust. Soc. Am. 92, 2756 (1992).

[8] I. Goldfarb, Z. Orenbach, I. Schreiber, F. Vafina, Shock Waves 7, 77 (1997).

[9] N. Mujica, S. Fauve, Phys. Rev. E 66, 021404 (2002).

[10] A. B. Wood, A Textbook of Sound, Bell (1944).

[11] N. T. Moxon, A. C. Torrance, S. B. Richardson, Appl. Acoust. 24, 193 (1988).

[12] K. B. Kann, Colloids Surf. A 263, 315 (2005).

[13] J. Ding, F.W. Tsaur, A. Lips, A. Akay, Phys. Rev. E 75, 041601 (2007).

[14] I. Ben Salem, R. M. Guillermic, C. Sample, V. Leroy, A.
Saint-Jalmes, B. Dollet, Soft Matter, 9, 1194 (2013).

[15] M. Minnaert, Phil. Mag. 16, 235 (1933).

[16] K. W. Commander, A. Prosperetti, J. Acoust. Soc. Am. 85, 732 (1989).

[17] V. Leroy, A. Strybulevych, J. H. Page, M. Scanlon, J. Acoust. Soc. Am. 123, 1931 (2008).

[18] J. Pierre, F. Elias, V. Leroy, Ultrasonics 53, 622 (2013).

[19] J. Pierre, R. M. Guillermic, F. Elias, W. Drenckhan, V. Leroy, Eur. Phys. J. E 36, 113 (2013).

[20] A. Maestro, W. Drenckhan, E. Rio, and R. Höhler, Soft Matter, 9, 2531 (2013).

[21] S. H. Lee, C. M. Park, Y. M. Seo, Z. G. Wang, C. K. Kim, Phys. Lett. A 373, 4464 (2009).

[22] see for instance chapter 4 in L. E. Kinsler, A. R. Frey, A. B. Coppens, J. V. Sanders, Fundamental of Acoustics, John Wiley \& Sons (2000).

[23] I. Cantat, Phys. Fluids 25, 031303 (2013).

[24] H. M. Princen, J. Colloid Interface Sci. 105, 150 (1985).

[25] N. Fang, D. Xi, J. Xu, M. Ambati, W. Srituravanich, C. Sun, X. Zhang, Nature Materials 5, 452 (2006).

[26] M. Yang, G. Ma, Z. Yang, P. Sheng, Phys. Rev. Lett. 110, 134301 (2013).

[] This is an approximate formula for $q$, see discussion in SM1 and J. G. H. Joosten, J. Chem. Phys. 80, 2363 (1984), Y. Couder, J. M. Chomaz, M. Rabaud, Physica D 37, 384 (1989), P. Sens, C. Marques, J. F. Joanny, Langmuir 9, 3212 (1993)

[] A movie showing the oscillations of the film and the ring is available in SM2. 